\title{
Hydrogen-Maser Principles and Techniques
}

\section{Citation}

Kleppner, Daniel, Howard C. Berg, S. B. Crampton, Norman F. Ramsey, Robert F. C. Vessot, H. E. Peters, and Jacques Vanier. 1965. Hydrogen-maser principles and techniques. Physical Review 138(4A): A972-A983.

\section{Published Version}

http://dx.doi.org/10.1103/PhysRev.138.A972

\section{Permanent link}

http://nrs.harvard.edu/urn-3:HUL.InstRepos:3203709

\section{Terms of Use}

This article was downloaded from Harvard University's DASH repository, and is made available under the terms and conditions applicable to Other Posted Material, as set forth at http:// nrs.harvard.edu/urn-3:HUL.InstRepos:dash.current.terms-of-use\#LAA

\section{Share Your Story}

The Harvard community has made this article openly available.

Please share how this access benefits you. Submit a story.

Accessibility 


\title{
Hydrogen-Maser Principles and Techniques*
}

\author{
D. Kleppner $\nmid \uparrow$ H. C. Berg $\ddagger$ S. B. Crampton,$\S$ and N. F. Ramsey \\ Harvard University, Cambridge, Massachusetts \\ AND
}

\author{
R. F. C. Vessot, H. E. Peters, and J. Vanier \\ Varian Associates, Beverly, Massachusetts
}

(Received 17 Decmeber 1964)

\begin{abstract}
Techniques and design principles relevant to the construction and operation of a hydrogen maser are presented in detail. These include methods for the generation of atomic hydrogen, state selection, design of the microwave cavity, production of very low magnetic fields, coating the hydrogen storage bulb, and tuning the maser. A figure of merit is introduced which indicates the optimum choice of parameters.
\end{abstract}

\section{INTRODUCTION}

A LTHOUGH the hydrogen maser has proved useful both as a spectroscopic tool and as a frequency standard, only a portion of its theory has so far been described. ${ }^{1}$ Most of the description of experiments with the maser have given the results but have omitted technical details. ${ }^{2-8}$ In the present paper, the interrelation of the various physical effects governing the maser's behavior will be discussed along with relevant operational and technical considerations.

The hydrogen maser operates between the groundstate hyperfine levels of atomic hydrogen. For use as a frequency standard, the maser oscillates on the transition $\left(F=1, m_{F}=0\right) \rightarrow\left(F=0, m_{F}=0\right)$ at a frequency of approximately $1420 \mathrm{Mc} / \mathrm{sec}$. Figure 1 is a schematic diagram. Molecular hydrogen is dissociated in the

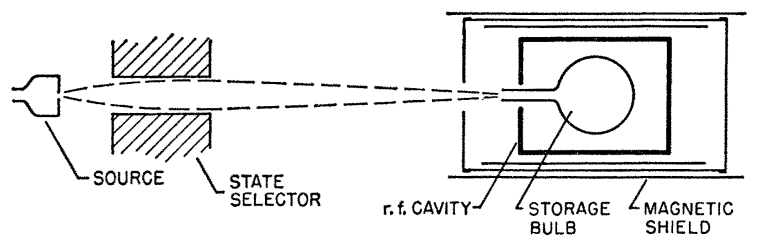

FIG. 1. Schematic diagram of the hydrogen maser.

*Work supported by the National Science Foundation and the Office of Naval Research (Harvard) and by the National Aeronautics and Space Administration and the Office of Naval Research (Varian).

$\dagger$ Alfred P. Sloan Foundation Fellow.

I Junior Fellow, Society of Fellows, Harvard University.

\$ National Science Foundation Postdoctoral Fellow.

iD. Kleppner, H. M. Goldenberg, and N. F. Ramsey, Phys. Rev. 126, 603 (1962).

${ }^{2}$ H. M. Goldenberg, D. Kleppner, and N. F. Ramsey, Phys.

Rev. Letters 5, 361 (1960); Appl. Opt. 1, 55 (1962).

${ }^{3}$ S. B. Crampton, D. Kleppner, and N. F. Ramsey, Phys. Rev, Letters 11, 338 (1963).

${ }^{4}$ H. C. Berg, D. Kleppner, and N. F. Ramsey, Bull. Am. Phys. Soc. 8, 379 (1963).

${ }^{5}$ S. B. Crampton, D. Kleppner, and H. G. Robinson, Bull. Am. Phys. Soc. 8, 351 (1963).

${ }^{6}$ S. B. Crampton and D. Kleppner, Bull. Am. Phys. Soc. 9, 451 (1964).

${ }^{7}$ H. G. Robinson, H. C. Berg, and S. B. Crampton, Bull. Am. Phys. Soc. 9, 564 (1964).

${ }^{8}$ E. N. Fortson, D. Kleppner, and N. F. Ramsey, Phys. Rev. Letters 13, 22 (1964). source and is formed into an atomic beam which passes through a state-selecting magnet. The emergent beam contains only atoms in the states $(F=1, m=1)$ and $(F=1, m=0)$. The beam passes into a storage bulb which has a specially prepared surface and in which the atoms remain for approximately $0.3 \mathrm{sec}$ before escaping. The bulb is located in a cavity tuned to the hyperfine transition frequency. Stimulated emission occurs if the beam flux is sufficiently high and a signal is produced in the cavity. This signal is detected by means of a small coupling loop. The cavity is surrounded by magnetic shields to reduce the ambient field and a small uniform field is produced at the storage bulb by a solenoid.

In Sec. II, some formulas are presented which govern the choice of design parameters. Subsequent sections are: III. Source, IV. State Selector, V. Vacuum System, VI. Cavity, VII. Magnetic Shields, VIII. Storage Bulb, IX. Electronics, and X. Tuning Methods.

\section{OPERATING CONDITIONS}

Basic formulas governing the operation of the hydrogen maser have been published by Kleppner, Goldbenberg, and Ramsey ${ }^{1}$ hereafter referred to as (KGR). Their results will be extended here in order to illustrate more clearly the interdependence of the design parameters. In particular, by including the effect of spin-exchange relaxation in the governing equations, it will be shown that for oscillation to occur on the transition $(F=1, m=0) \rightarrow(F=0, m=0)$ there is not only a minimum beam flux but also a maximum permissible flux. The analysis leads to a constraint condition governing the cavity geometry, storage-bulb size, and various relaxation times. Unless the constraint is satisfied, the maser will not oscillate, and the constraint equation leads to a useful figure of merit which predicts how suitable a given configuration will be.

Our starting point is KGR Eq. (7),

$$
P=\frac{1}{2} \operatorname{Ih} \omega \frac{x^{2}}{\left(1 / T_{b}\right)^{2}+x^{2}+\left(\omega-\omega_{0}\right)^{2}},
$$

where $P$ is the power radiated by the atoms, $I$ is the 
net input flux, i.e., the difference in the flux of atoms entering in the state $(F=1, m=0)$ and the state $(F=0, m=0), \omega_{0}$ is the resonance frequency, and $x=\mu_{0} H_{z} / \hbar$, where $H_{z}$ is the oscillating field amplitude. $T_{b}$ is the mean storage time of the bulb (denoted by $\gamma^{-1}$ in KGR). Under the assumptions discussed below, this equation can be generalized to the case in which other relaxation processes occur. In particular, we shall distinguish between processes which relax the population difference between the two states of interest and those which relax the oscillating moment. In analogy with $\mathrm{nmr}$ terminology, we designate the decay times for the processes by $T_{1}$ and $T_{2}$, respectively. Then it can be shown that Eq. (1) becomes ${ }^{9,10}$

$$
P=\frac{1}{2} I h \omega \frac{x^{2}}{1 /\left(T_{1} T_{2}\right)+x^{2}+\left(T_{2} / T_{1}\right)\left(\omega-\omega_{0}\right)^{2}} .
$$

If the only relaxation mechanism is escape of atoms from the storage bulb, then $T_{1}=T_{2}=T_{b}$. However, we must also allow for hydrogen-hydrogen spin exchange (s.e.). For this process, $T_{2}=2 T_{1},{ }^{11,12}$ where

$$
\left(1 / T_{1}\right)_{\mathrm{see.}}=n \sigma \bar{v}_{r} \text {. }
$$

Here $\sigma$ is the hydrogen "spin-flip" cross section, ${ }^{13}$ estimated by $\mathrm{Mazo}^{14}$ to be $2.85 \times 10^{-15} \mathrm{~cm}^{2}, \bar{v}_{r}$ is the average relative hydrogen velocity $\left[\bar{v}_{r}=4(k T / \pi m)^{1 / 2}\right.$ $=3.58 \times 10^{5} \mathrm{~cm} / \mathrm{sec}$ at $\left.T=308^{\circ} \mathrm{K}\right]$, and $n$ is the hydrogen density given by

$$
n=I_{\text {tot }} T_{b} / V_{b}
$$

where $I_{\text {tot }}$ is the total flux of atoms entering the storage bulb, and $V_{b}$ is the storage-bulb volume. Normally, the states $(F=1, m=0)$ and $(F=1, m=1)$ are focused, so that $I_{\text {tot }}=2 I$. If the state selection is imperfect, other states may be present so that $I_{\text {tot }} / I$ can have a large value.

There are a variety of other possible relaxation processes, ${ }^{11}$ some of which are discussed in KGR. For these, $T_{1}$ and $T_{2}$ are not in general the same. We shall allow for them by letting $T_{1}{ }^{\prime}$ and $T_{2}{ }^{\prime}$ stand for the total relaxation times due to all processes other than escape from the bulb and hydrogen spin exchange. Then

$$
\begin{aligned}
& 1 / T_{1}=1 / T_{1}{ }^{\prime}+\left(1 / T_{1}\right)_{\text {s.e. }}+1 / T_{b}, \\
& 1 / T_{2}=1 / T_{2}{ }^{\prime}+\left(1 / T_{2}\right)_{\text {s.e. }}+1 / T_{b} .
\end{aligned}
$$

Here the subscript s.e. indicates that the relaxation mechanism is spin exchange.

We assume, for the present, that $T_{1}{ }^{\prime}$ and $T_{2}{ }^{\prime}$ are

${ }^{9}$ S. B. Crampton, Ph.D. thesis, Harvard, 1964 (unpublished). ${ }^{10}$ P. L. Bender, Phys. Rev. 132, 2154 (1963).

${ }^{11}$ H. C. Berg, Ph.D. thesis, Harvard, 1964 (unpublished). Also H. C. Berg, Phys. Rev. 137, A1621, (1965).

${ }^{12}$ J. P. Wittke and R. H. Dicke, Phys. Rev. 103, 620 (1956)

${ }^{13}$ L. C. Balling, R. J. Hanson, and F. M. Pipkin, Phys. Rev. 132, 2154 (1964).

${ }^{14}$ R. M. Mazo, J. Chem. Phys. 34, 169 (1961). constants, insofar as they do not depend on the hydrogen atom flux or on the oscillation level of the maser. This is not necessarily true, since the structure of the storage-bulb wall may depend on the hydrogen density and the state populations, as will be discussed below.

To analyze the conditions for stationary oscillation of the maser, we proceed as in KGR by equating the radiated power to the dissipated power $\omega W / Q$ where $W$ is the stored energy in the cavity. By substituting Eqs. (3), (4), and (5) in Eq. (2) with $\omega=\omega_{0}$ and rearranging, using $x^{2}=\left(\mu_{0} / \hbar\right)^{2}\left(8 \pi W / V_{c}\right) \eta$, we arrive eventually at the following relation between the power radiated and the beam flux:

$$
P / P_{c}=-2 q^{2}\left(I / I_{\mathrm{th}}\right)^{2}+(1-c q) I / I_{\mathrm{th}}-1 .
$$

$P_{c}$ is defined by the following:

where

$$
P_{c}=\omega \hbar^{2} V_{c} / 8 \pi \mu_{0}^{2} Q n T_{t}^{2},
$$

$$
\begin{aligned}
1 / T_{t}{ }^{2} & =\left(1 / T_{1}{ }^{\prime}+1 / T_{b}\right)\left(1 / T_{2}{ }^{\prime}+1 / T_{b}\right), \\
\eta & =\left\langle H_{z}\right\rangle_{\text {bulb }}{ }^{2} /\left\langle H^{2}\right\rangle_{\text {cavity }},
\end{aligned}
$$

and

$I_{\text {th }}$ is defined by

$$
V_{c}=\text { cavity volume. }
$$

$$
I_{\mathrm{th}}=2 P_{c} / \hbar \omega .
$$

[Physically, $I_{\text {th }}$ is the net threshold flux for oscillation to occur providing spin exchange can be neglected. In the presence of spin exchange, the net flux for oscillation is somewhat larger, and is given by $I_{\min }$, as shown in Eq. (13) below.]

Also,

$$
c=\left[\frac{1 / T_{b}+1 / T_{1}^{\prime}}{1 / T_{b}+1 / T_{2}^{\prime}}\right]^{1 / 2}+2\left[\frac{1 / T_{b}+1 / T_{2}{ }^{\prime}}{1 / T_{b}+1 / T_{1}^{\prime}}\right]^{1 / 2} .
$$

The quantity $q$ is an important quality parameter which has the following value:

$$
q=\frac{\sigma \bar{v}_{r} \hbar}{8 \pi \mu_{0}^{2}} \frac{T_{b}}{T_{t}} \frac{V_{c}}{\eta V_{b}} \frac{1}{Q} \frac{I_{\text {tot }}}{I} .
$$

From Eq. (6) it is apparent that the power radiated by the maser oscillator is a quadratic function of the beam flux. If we require that both the flux and the power be positive quantities, we obtain the following condition:

$$
q<(c-2 \sqrt{2}) /\left(c^{2}-8\right) .
$$

It also follows that the maximum and minimum net beam fluxes for oscillation are given by

$$
I_{\min } / I_{\text {th }}=\frac{1-c q \pm\left[1-2 c q+\left(c^{2}-8\right) q^{2}\right]^{1 / 2}}{4 q^{2}}
$$

Because of spin exchange, the resonance linewidth $\Delta \nu$ depends on the flux. It is given by the following 


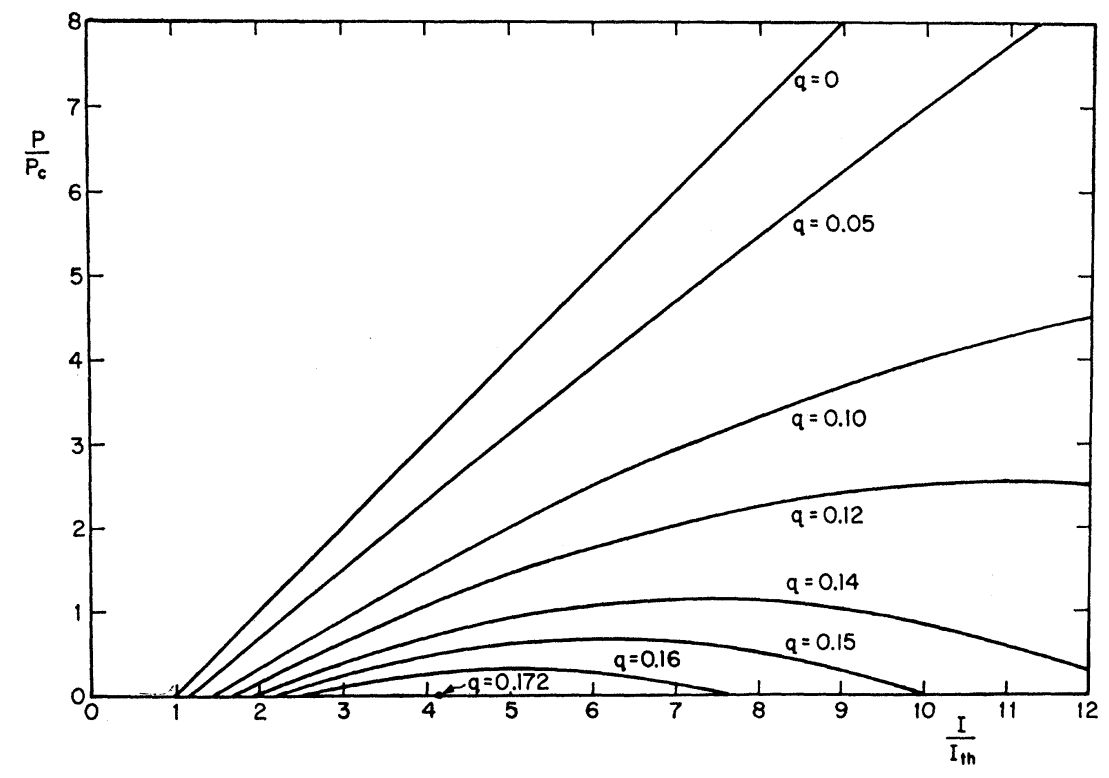

FIg. 2. $P / P_{c}$ versus $I / I_{\text {th }}$ for different values of the parameter $q$. This family of curves shows the strong influence of $q$ on the operating conditions. If spin exchange is neglected, $q=0$ and radiated power increases monatonically with beam flux. For $q>0$, there is an upper limit to the flux for oscillation to occur and above a certain value $(q=0.172)$ the maser cannot radiate at any beam flux. $q$ is defined by Eq. (11). expression :

$$
\Delta \nu=1 / \pi T_{2}=(1 / \pi)\left[\left(1 / T_{2}{ }^{\prime}+1 / T_{b}\right)+\left(I / I_{\mathrm{th}}\right) q / T_{t}\right] .
$$

To proceed further, we must choose a particular ratio for $T_{1}{ }^{\prime}$ and $T_{2}{ }^{\prime}$. In the event that $T_{1}{ }^{\prime}=T_{2}{ }^{\prime}, c=3$, and we have

$$
\begin{aligned}
P / P_{c} & =-2 q^{2}\left(I / I_{\mathrm{th}}\right)^{2}+(1-3 q) I / I_{\mathrm{th}}-1, \\
q<3-2 \sqrt{2} & =0.172 \\
I_{\min } / I_{\mathrm{th}} & =\frac{1-3 q \pm\left(1-6 q+q^{2}\right)^{1 / 2}}{4 q^{2}} \\
\Delta \nu & =\left(1 / \pi T_{t}\right)\left(1+q I / I_{\mathrm{th}}\right) .
\end{aligned}
$$

For purposes of tuning, to be discussed in Sec. X, it is essential that the ratio of the resonance widths at the extremes of permissible flux be large. This ratio is

$$
r=\frac{\Delta \nu \max }{\Delta \nu \min }=\frac{1+q+\left(1-6 q+q^{2}\right)^{1 / 2}}{1+q-\left(1-6 q+q^{2}\right)^{1 / 2}} .
$$

To illustrate the behavior of the maser as the factor $q$ varies, Eq. (15) is plotted in Fig. 2 for a few values of $q$. Equations (17) and (19) are plotted in Fig. 3, which shows both $r$ and the permissible extremes of flux as a function of $q$. It is evident from the figures that a relatively small decrease in $q$ can take the maser from a state of critical dependence on flux, or even no oscillation at all, to a favorable region of oscillation.

In order for the maser to operate as an oscillator, it is essential that the inequality in (16) be satisfied. If we substitute Eq. (11) in Eq. (16), we obtain

$$
T_{b} / T_{t} \times V_{c} /\left(\eta V_{b}\right) \times 1 / Q \times I_{\text {tot }} / I<3.47 \times 10^{-4} .
$$

As an example, let us insert the following typical values:

$$
V_{c} / V_{b}=6.7, \eta=3, I_{\text {tot }} / I=2, Q=3 \times 10^{4} .
$$

Then, for oscillation,

$$
T_{b} / T_{t}=1+T_{b} / T_{1}<2.32 \text {. }
$$

If unwanted relaxation mechanisms are present, the bulb storage time must be decreased so that this inequality holds. Conversely, if a given bulb geometry and storage time are required, Eq. (21) can be used to set an upper limit on permissible relaxation.

If we assume $V_{c}=1.3 \times 10^{4} \mathrm{~cm}^{3}, T_{b} / T_{t}=1.3$, and $T_{b}=0.3 \mathrm{sec}$, reasonable values in practice, then we have further

$$
\begin{aligned}
P_{c} & =5.9 \times 10^{-13} \mathrm{~W}, \\
I_{\mathrm{th}} & =1.3 \times 10^{12} \text { particles } / \mathrm{sec}, \\
q & =0.097 \\
I_{\min } & =1.9 \times 10^{12} \text { particles } / \mathrm{sec}, \\
r & =4 .
\end{aligned}
$$

and

The time $T_{2}$ can be measured by observing the decay of radiation when the maser is pulsed while operating below oscillation threshold. II In this condition, spinexchange is usually negligible, so that if the relation between $T_{1}^{\prime}$ and $T_{2}{ }^{\prime}$ is known, $T_{t}$ can be determined from Eq. (8). The other quantities involved in $q$ can be calculated in principle, though the quantity $\eta$ is difficult to compute precisely, except for the case of simple geometry, such as a cylindrical bulb in a cylindrical cavity, which will be discussed in Sec. VIII. Alternatively, the cavity $Q$ can be decreased by increasing the coupling until the maser ceases to operate at any flux. At this point, the inequality (12) becomes an equality. By measuring $Q$ at this cutoff condition and 

and $I_{\min } / I_{\text {th }}$ versus $q$. The maser can oscillate if the flux obeys $I_{\min } \leqslant I \leqslant I_{\max }$, indicated by the shaded region. The curve $r$ gives the ratio $I_{\max } / I_{\min }$ and is a useful measure of the amount by which the resonance line can be broadened by spin exchange.
FIG. 3. $I_{\max } / I_{\text {th }}$

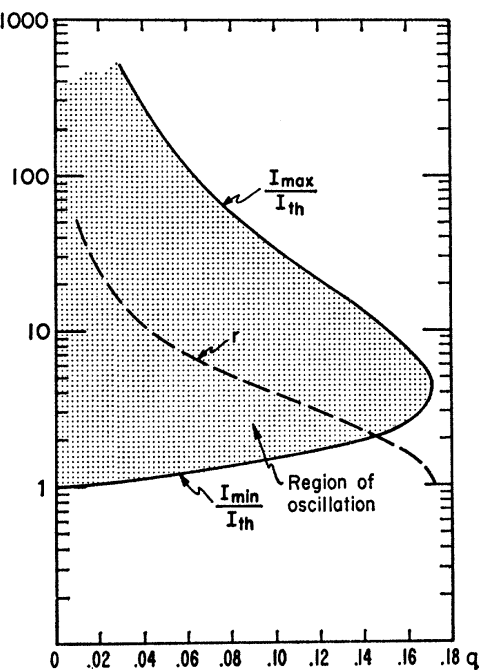

at the normal operating condition, $q$ can be determined for the latter case.

It should be pointed out that care must be used in applying this analysis. In some cases, $T_{1}$ is known once $T_{2}$ has been measured. For instance, in the case of spinexchange collisions between hydrogen and a spin- $-\frac{1}{2}$ or spin-1 background gas, $T_{2}=\frac{4}{3} T_{1}{ }^{11}$ However, unless the relaxation mechanisms are understood, the ratio of $T_{1}$ to $T_{2}$ must be separately measured. Any mechanism which converts atoms to molecular form in effect behaves like a hole through the wall and decreases the storage time of the bulb $T_{b}$. In addition, two assumptions have been made which do not necessarily always hold. In the first place, the maser has been treated as a simple two-level system. This is not necessarily true if there is coupling with the remaining two hyperfine levels. As an example, relaxation due to motion of the atoms through an inhomogeneous magnetic field couples the three upper hyperfine states. The effect of this is, on the one hand, to relax the oscillating moment, and on the other hand, to feed atoms from the $(1,1)$ and $(1,-1)$ states into the $(1,0)$ state as this is depleted by radiation to the ground state. A full analysis of this can be carried out using the density matrix formalism, ${ }^{9}$ but for many purposes the process can be neglected by ensuring that magnetic relaxation is not large.

The second assumption is that the remaining relaxation processes are simply characterized by constant rates. In fact, there is evidence to indicate that part of the relaxation at the storage-bulb surface can occur at a rate proportional to the hydrogen density. ${ }^{11}$ One effect of such a process is the enhancement of the value of $\sigma$ entering Eq. (1), and this must be taken into account.

\section{SOURCE}

Of the three time-honored devices for dissociating molecular hydrogen-Wood's discharge, rf (or microwave) discharge, and thermal dissociator-the rf discharge has so far proven to be the most convenient for use in the hydrogen maser. The Wood's tube is cumbersome to build and operate, and the thermal dissociator produces a relatively hot beam which is difficult to focus. A simple rf discharge is described here.

The discharge takes place in a spherical Pyrex bulb, approximately $2 \frac{1}{2} \mathrm{~cm}$ diam. The source aperture is a hole typically $\frac{1}{2} \mathrm{~mm}$ diam, though holes up to $1 \mathrm{~mm}$ diam function satisfactorily. The aperture can be connected to the bulb by a short length of Pyrex tubing or led directly through the wall of the bulb, as shown in Fig. 4. If desired, the flux to the source chamber pump can be reduced by using a multitube glass collimator instead of a simple hole. ${ }^{15-17}$ Pressure in the discharge tube is typically $0.3 \mathrm{~mm} \mathrm{Hg}$, though the discharge operates well at pressures between 0.05 and $0.8 \mathrm{~mm} \mathrm{Hg}$. Power for the discharge is supplied by a single-tube oscillator operating at a frequency of 200 $\mathrm{Mc} / \mathrm{sec}$ or higher. A variable link is used to couple the power to the discharge. A circuit for the oscillator and details of the coupling link are shown in Fig. 5. Tuning is facilitated by using a directional power meter in the transmission line. Matching must be done with the tube lit. Under typical conditions the oscillator tube will draw $150 \mathrm{~mA}$ at $400 \mathrm{~V}$, and the input power to the discharge tube is $10 \mathrm{~W}$. At higher power levels, the discharge tube should be cooled with forced air.

It is possible to treat the discharge tube walls to reduce recombination using phosphoric acid or Dri Film $^{18}$ (but not Teflon, which is decomposed by the discharge). The most satisfactory operation has been

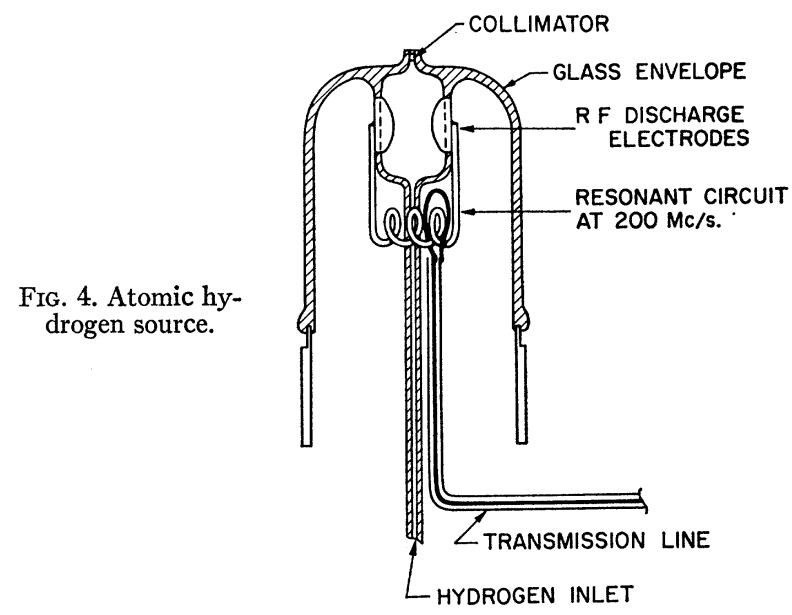

${ }^{15}$ R. F. C. Vessot and H. E. Peters, IRE (Inst. Radio Engrs.), Trans. Instr. 11, 183 (1962); J. Vanier, H. E. Peters, and R. F. C. Vessot, $i b i d$. (to be published).

${ }_{16} \mathrm{P}$. Grivet and N. Bloembergen, Quantum Electronics III (Columbia University Press, New York, 1964), pp. 333-347; also pp. 409-417.

17 The collimators described in Refs. 16 and 17 are effective at low source pressures but are not useful if high flux is desired. Finer collimators which are useful over a wider pressure range can be supplied by Permeonics, Inc., Southbridge, Massachusetts.

${ }_{18}$ Type SC-02 (dimethyldichlorosilane) General Electric Company, Silicone Products Department, Waterford, New York. 


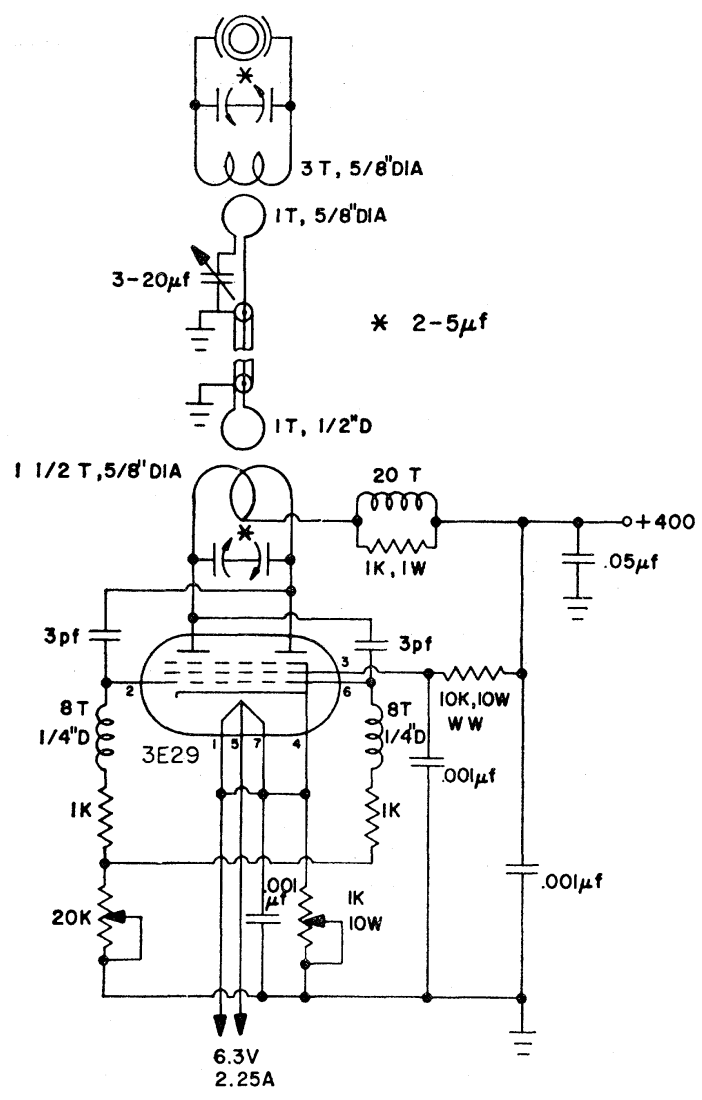

FIG. 5. Hydrogen discharge power oscillator.

obtained with untreated Pyrex. Normally, the tube must age for a few hours before the discharge acquires its characteristic red color. After long operation, the discharge tube becomes discolored by the decomposition of the glass; black borosilicate products appear on the inside surface of the tube. This does not appear to have a serious effect on the operation of the discharge.

Hydrogen may be introduced to the discharge by means of a conventional gas-handling system or through a combination purifier, pressure reducer, and flow controller consisting of a simple palladium leak. (Conventional regulators with variable leaks are difficult to reset and usually will not regulate pressure to better than $10 \%$.) Both the leak and a servo system for controlling the source pressure are shown in Fig. 6. The source pressure is monitored by a Pirani gauge consisting of a thermistor bead suspended in the hydrogen inlet line. The gauge is housed in a thermally controlled box which also houses the resistance bridge and servo preamplifier. Flux through the palladium leak is controlled by varying its temperature with a heating coil.

\section{STATE SELECTOR}

The hexapolar field proposed by Friedburg and Paul ${ }^{19}$ is ideally suited to the hydrogen maser. This type of state selector has a large acceptance angle, but in general yields an atomic beam of relatively large area. Fortunately, the entrance to the storage bulb can be made large enough to accept a substantial part of the beam.

The design of a simple permanent six-pole magnet has been given by Christensen and Hamilton. ${ }^{20} \mathrm{~A}$ magnet similar to their design has been constructed and has the following properties: gap diameter, $\frac{1}{8}$ in.; length, 3 in. ; maximum field at pole tip (as measured by a rotating coil magnetometer), 9900 Oe. The magnet can be bolted or glued together with epoxy and is magnetized by passing approximately $500 \mathrm{~A}$ through 33 turns of wire wound on the poles or by using a pulsed magnetizer. ${ }^{21}$

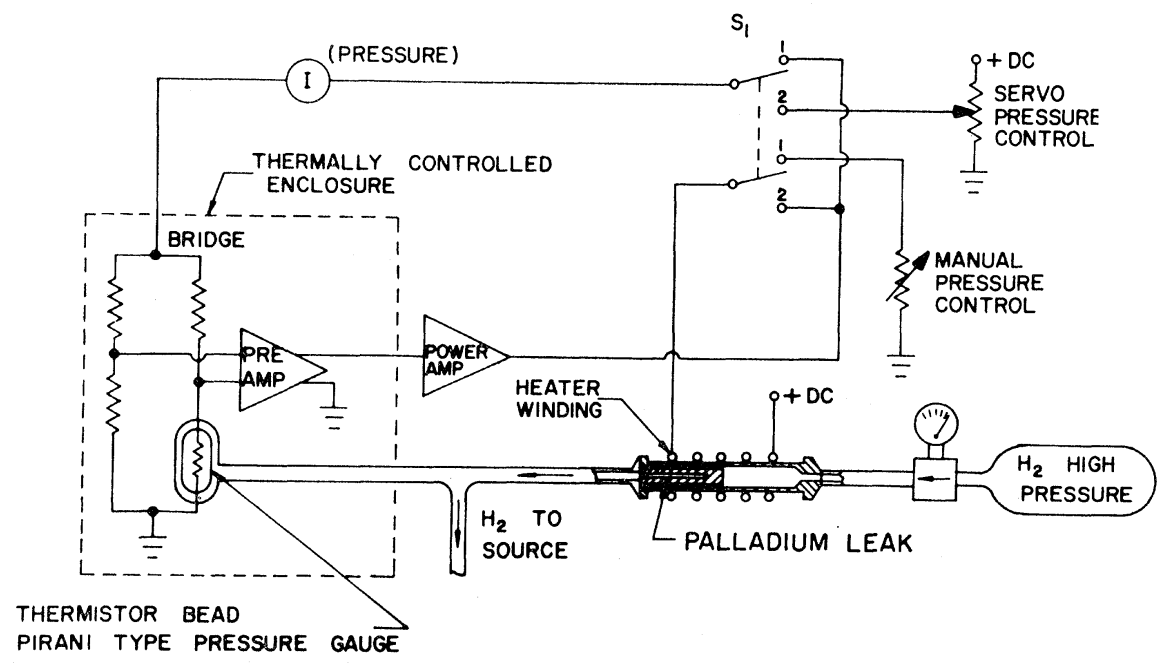

FIG. 6. Hydrogen gas-handling system and flow regulator. The palladium leak is made from an alloy pellet $70 \%$ palladium, $30 \%$ silver, approximately $\frac{1}{2}$-in. long, $\frac{3}{16}$-in. o.d., $\frac{1}{16}$-in. i.d. The Piranigauge preamplifier and bridge are similar to those in Fig. 8.

${ }^{19} \mathrm{H}$. Friedburg and W. Paul, Naturwiss. 38, 159 (1951).

${ }^{20}$ R. L. Christensen and D. R. Hamilton, Rev. Sci. Instr. 30, 356 (1959).

${ }^{21}$ These magnets can be obtained commercially from Varian Company, Bomac Division, Beverly, Massachusetts. 


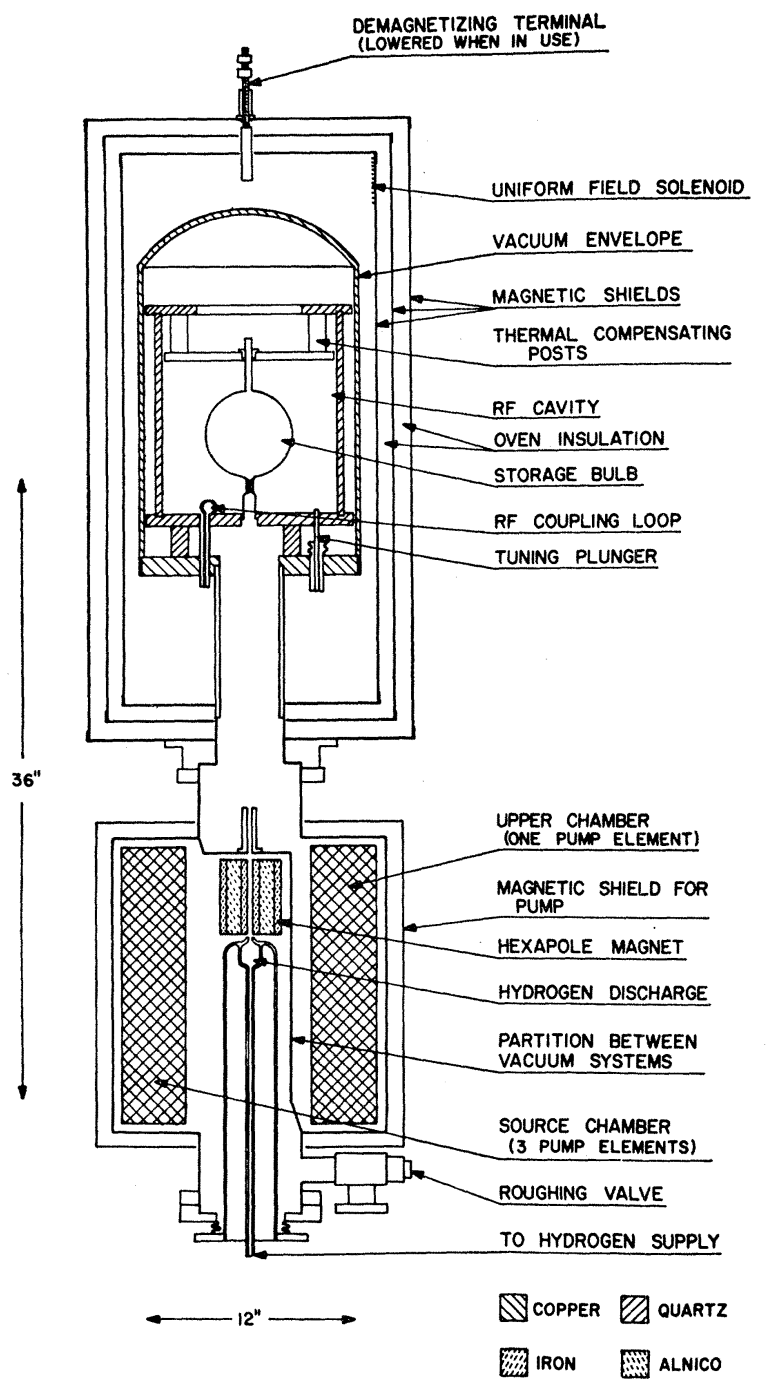

FIG. 7. Details of hydrogen maser.

Typical dimensions for the beam optics of a hydrogen maser are indicated in Fig. 7. The total flux from a collimated source is $10^{16} \mathrm{atom} / \mathrm{sec}$ with a source pressure of $0.1 \mathrm{~mm} \mathrm{Hg}$. The collimater is composed of 400 glass tubes forming a cylinder $1 \mathrm{~mm}$ diam and $1 \mathrm{~mm}$ long. The net flux of atoms to the bulb in the desired state is approximately $2 \times 10^{12}$ atoms/sec.

\section{VACUUM SYSTEM}

Because of the possibility of contamination of the storage bulb surface, the hydrogen maser demands a relatively clean vacuum system. Mercury diffusion pumps are satisfactory, providing they are properly baffled. Oil diffusion pumps can lead to appreciable contamination of the surface, even when there is a very low residual pressure, and are not recommended. The most satisfactory pump appears to be an ion sputtering pump. These pumps have both high speed and high capacity for hydrogen. For example, a 75 liter/sec VacIon pump can absorb well over $1 \frac{1}{2}$ mole of $\mathrm{H}_{2}$, providing care is taken to prevent the cathode from shorting to the anode as it expands due to the absorbed hydrogen.

In general, it is desirable to pump differentially the source and storage bulb chambers in order to prevent excessive beam scattering. Fortunately, a relatively high background pressure of molecular hydrogen (up to $10^{-5} \mathrm{~mm} \mathrm{Hg}$ ) does not interfere with the maser's operation since $\mathrm{H}_{2}$ is a good buffer gas. The background pressure with the beam turned off should be $10^{-8} \mathrm{~mm}$ $\mathrm{Hg}$, or less, to prevent broadening of the resonance line due to spin exchange collisions with $\mathrm{O}_{2}$.

Conservative design of the vacuum system calls for a pumping speed of 200 liter/sec (air) for the source chamber, and perhaps half as much for the rest of the system. Under conditions of extremely high flux, the source chamber pressure will be $3 \times 10^{-5} \mathrm{~mm} \mathrm{Hg}$ (ion gauge, uncorrected for $\mathrm{H}_{2}$ ), while under normal operating conditions the pressure is a factor of 10 lower.

Figure 7 shows a system employing a titanium sputtering pump enclosing the source structure. Here, a VacIon 250-liter/sec pump is used with the modification that a partition is installed which allows one of the four pumping elements to pump the upper chamber. The state-selecting magnet is mounted on the partition. Flanges at the upper and lower ends of the pump connect to the bell jar and source assemblies, respectively. Stray fields from the pump magnets are reduced by a shield of $\frac{1}{16}$-in. Armco iron surrounded by a second shield of $\frac{1}{16}$-in. Mu Metal.

The microwave cavity must be evacuated to prevent atmospheric disturbance of the tuning. ${ }^{15,16}$ This may be done by a separate roughing system, or, as shown in Fig. 7, by connecting it directly to the high-vacuum system. To afford thermal isolation from the bell jar, the cavity supports are three thin-walled quartz tubes. The bell jar of $\frac{1}{4}$-in. copper provides a thermal enclosure for the cavity. The copper bell-jar base plate is connected to the pump manifold by a thin-walled stainless steel neck section to minimize heat transfer from the base plate to the maser frame. Further aspects of the thermal control of the cavity enclosure are dealt with in the next section.

\section{CAVITY}

Stability of the microwave cavity is critical to the operation of the maser. The problem is simply summarized by noting that the fraction of the atomic resonance linewidth by which the maser is "pulled" by a mistuned cavity is identical to the fraction of the cavity linewidth by which the cavity is mistuned. This is apparent from the following expression for the shift of the oscillator frequency $\nu$, from the true resonance frequency $\nu_{0}$, by a cavity tuned to a frequency $\nu_{c}{ }^{1}$

$$
\frac{\nu-\nu_{0}}{\nu_{0}}=\frac{\nu_{c}-\nu_{0}}{\nu_{0}} \frac{\Delta \nu_{r}}{\Delta \nu_{c}} \text {, }
$$


where $\Delta \nu_{r}$ and $\Delta \nu_{c}$ are the resonance and cavity linewidths, respectively.

In practice, the major source of drift of the maser is frequency pulling due to cavity drift. Although a particular cavity design is described here, it should be emphasized that other approaches are possible. In particular, it should be possible to stabilize the cavity frequency with a servo system. However, at the time of writing, such a system has not been put into operation.

The most convenient cavity mode is the cylindrical $T E_{011}$ mode, since this has only azimuthal wall currents so that good contact between the cylinder and end plates is not required. The corresponding spherical mode should also be favorable, though it is difficult to construct.

The electrical properties of the $T E_{011}$ mode are well described in the literature, ${ }^{22}$ and only a few points will be summarized here. The unloaded cavity resonates with length and diameter both equal to $27.6 \mathrm{~cm}$. The theoretical $Q$ is 87000 for silver-plated walls. In practice, $Q=60000$ is obtainable. The quartz storage bulb does not appreciably affect $Q$, but a bulb $15 \mathrm{~cm}$ in diameter with walls $1 \mathrm{~mm}$ thick will decrease the resonant length by about $5 \mathrm{~cm}$.

Power is coupled from the cavity by a loop mounted in an end plate near the position of maximum magnetic field. A loop area $\approx 1 \mathrm{~cm}^{2}$ will couple the cavity critically to a $50-\Omega$ line.

Since thermal and mechanical stability are of great importance, it is desirable to make the cavity tube out of quartz. This is done commercially by grinding quartz pipe to the correct i.d. and then fusing a silver film to the inner surface. ${ }^{23} \mathrm{~A}$ wall thickness of $\frac{5}{16} \mathrm{in}$. gives adequate mechanical strength, while the silver coating should be at least 0.001 in. to ensure minimum wall loss.

Because loading by the storage bulb changes the cavity length appreciably, the position of one end plate must be adjustable. For maximum stability the end plate should be mounted rigidly on spacers which are cut to bring the cavity within the range of a fine tuning control. For short term experiments involving frequent storage bulb changes, it is convenient to mount the end plate on a threaded drum with at least a 6-in. diam and a micrometer thread. After adjustment the end plate must be clamped in position.

Fine tuning can be accomplished by a tuning plunger or by coupling reactance to the cavity. A $\frac{1}{4}$-in.-diam plunger protruding through the end plate near the electric field maximum has a tuning sensitivity of approximately $7 \mathrm{kc} / \mathrm{sec} / \mathrm{in}$. The tuning range of the plunger is limited to about $70 \mathrm{kc} / \mathrm{sec}$ by the fact that it eventually couples in the $T M_{111}$ mode. Other plungers have a tuning coefficient roughly proportional to their cross-sectional areas.

${ }^{22}$ C. G. Montgomery, Techniques of Microwave Measurement (McGraw-Hill Book Company, Inc., New York, 1947), p. 297.

${ }^{23}$ Syncor Products Company, Malden, Massachusetts.
Reactance tuning is a very convenient fine-tuning method, since it is accomplished without mechanical adjustment. If the load coupled to the cavity by a line of impedance $Z_{0}$ is varied from $Z_{0}$ (i.e., a matched load) to $Z_{0}(1+j X)$, where $X \ll 1$, then the cavity will be detuned by an amount $\delta v_{c}$ given by ${ }^{24}$

$$
\delta \nu_{c}=(-\beta / 2 Q) X \text {. }
$$

$\beta$ is the coupling coefficient. ${ }^{25} \mathrm{~A}$ simple method for introducing the reactance is by coupling a fraction of the power from the cavity line with a directional coupler whose output is terminated by a crystal diode or a varactor. The reactance of the diode is adjusted by a biasing current. Although this presents a resistive as well as a reactive mismatch to the line, the coupling is sufficiently small so that the resultant power loss is negligible.

Preliminary tuning of the cavity to an accuracy of about $500 \mathrm{cps}$ can be accomplished by conventional reflection techniques, providing care is taken not to vary the load reactance coupled to the cavity during the process. Final tuning is done with the maser itself, as described in Sec. X.

In general, it is necessary to regulate thermally the cavity to limit drift. Thermal sensitivity of the cavity can be reduced by mounting an end plate on metal spacers with a length chosen to shorten the cavity the necessary amount to compensate for the effect of expansion of the cylinder. A typical thermal coefficient for a partially compensated cavity is $1 \mathrm{kc} / \mathrm{sec}{ }^{\circ} \mathrm{C}$.

The system in Fig. 7 uses a cavity designed to provide as much thermal contact as possible among its components in order to reduce the effect of thermal gradients. Insofar as possible, the cavity is thermally isolated from the base structure. The base structure and bell jar assembly form an isothermal enclosure which is mounted so as to provide as little heat conduction as possible to the lower manifold. The bell jar is enclosed in an oven by a thermally insulated aluminum cylinder nesting outside the inner magnetic shield. A second similar oven encloses the first. Thermal control of the two ovens and the neck temperature results in the control of the bell jar temperature to about $0.01^{\circ} \mathrm{C}$. Sensing and temperature correction are provided by the circuit shown in Fig. 8.

A word of caution about materials. Due to the requirements for very low magnetic fields, discussed in the next section, great care must be taken to avoid any ferromagnetic materials in the cavity. Stainless steels should not be used since at machined edges magnetically hard spots develop which cannot be removed by annealing. Even nickel plating over a nonmagnetic base material can cause difficulty. All cavity parts should be checked for magnetism during assembly.

${ }^{24}$ Reference 22, p. 291.

${ }^{25}$ E. L. Ginzton, Microwave Measurements (McGraw-Hill Book Company, Inc., New York, 1957), p. 290. 
FIG. 8. Temperature sensing and servo systems.

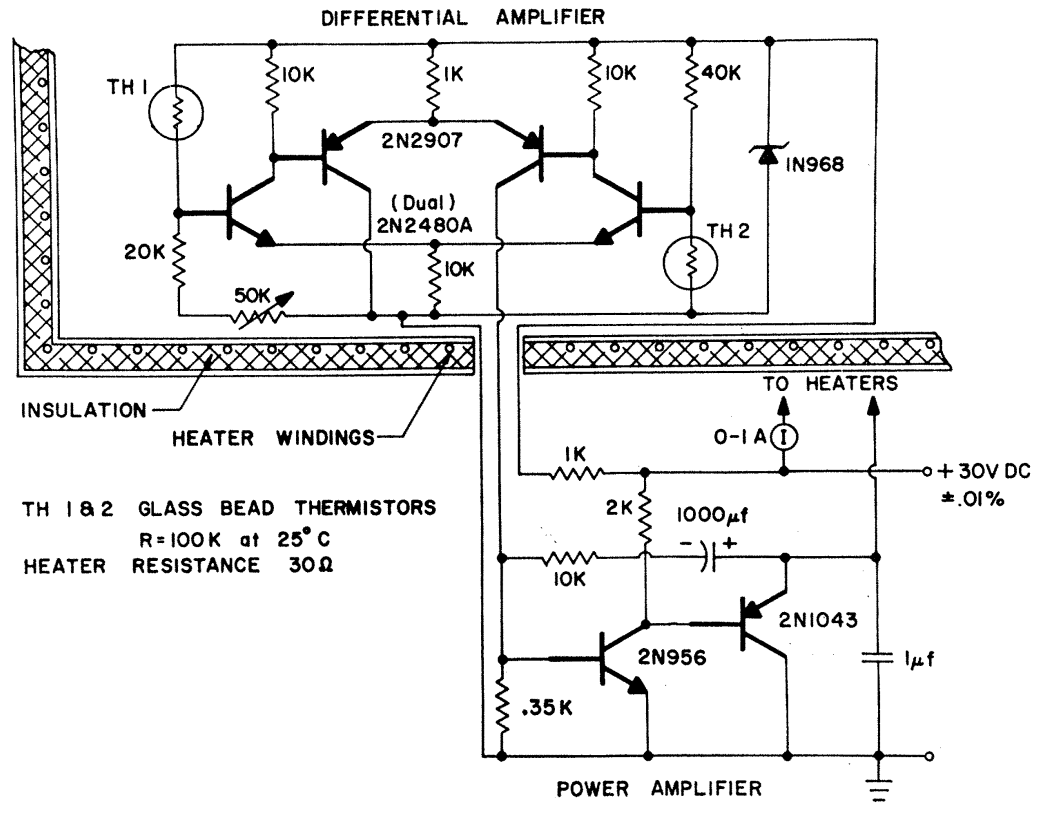

\section{MAGNETIC SHIELDS}

As a result of the quadratic field dependence of the transition $(F=1, m=0) \rightarrow(F=0, M=0)$, it is desirable to reduce the ambient field at the storage bulb to a very low value, preferably $1 \mathrm{mOe}$ or less. The most satisfactory way to accomplish this is through the use of magnetic shields. Ideally, the shields completely eliminate the magnetic field, and then a small uniform field is applied with a solenoid.

In practice, the low-field limit at which the maser operates is determined by either (a) the magnitude of the residual field which may lie in an undesirable direction, or (b) the gradients in the residual field which can cause prohibitive relaxation as the Zeeman frequency is reduced. In the first case, the applied field must be large enough so that the oscillating moment is substantially parallel to the oscillating field. However, even then, owing to (b), gradients of the residual transverse field can cause relaxation owing to the random motion of the atoms. As discussed in KGR, this relaxation rate is uniform at low fields and drops rapidly when the Zeeman frequency exceeds the mean "rattle frequency" of the atoms in the bulb. Consequently, large gradients necessitate a relatively large uniform field. In poorly demagnetized shields, this means a field of 5 mOe or more.

The magnetic field dependence of the transition of interest is

$$
\nu=\nu_{0}+2750 H^{2} \mathrm{cps},
$$

where $H$ is the applied field in oersted.

The fractional shift of the frequency due to a small change in field is

$$
\delta \nu / \nu=3.9 \times 10^{-6} H^{2}(\Delta H / H) .
$$

The maser has been operated at fields as low as $6 \times 10^{-5}$ Oe, where field stability is no longer a significant problem.

In principle, it is possible to shield external fields to a high degree by the use of successive concentric shields. For the case of a uniform field at right angles to a series of three infinitely thin concentric cylinders, the shielding factor, i.e., the ratio of the applied external field $H_{0}$ to the net internal field $H_{i}$, is given ${ }^{25 a}$ by

$$
\frac{H_{0}}{H_{i}}=\frac{1}{2} \frac{\mu_{1} t_{1}}{r_{1}} \frac{\mu_{2} t_{2}\left(s_{12}-s_{12}{ }^{2} / 2 r_{2}\right)}{r_{2}{ }^{2}} \frac{\mu_{3} t_{3}\left(s_{23}-s_{23}{ }^{2} / 2 r_{3}\right)}{r_{3}{ }^{2}},
$$

where $\mu$ is the permeability of the shields, and the dimensions are as shown in Fig. 9. It is apparent from this that with moderately high permeability, very high shielding factors are obtainable.

A shield configuration which has proven successful in use consists of the following: The shields are composed of three coaxial cylinders fabricated from 0.025 -in. Moly Permalloy. The innermost is 14 in. in diameter and $30 \mathrm{in}$. long; the middle shield is $16 \mathrm{in}$. in diameter and $32 \mathrm{in.} \mathrm{long;} \mathrm{and} \mathrm{the} \mathrm{outer} \mathrm{shield} \mathrm{is} 18 \mathrm{in}$. in diameter and $36 \mathrm{in}$. long. The seams are spot-welded over $1 \frac{1}{2}$-in. laps. (Mu Metal has also been used but is not as satisfactory as Moly Permalloy.) The inner two shields have end caps spun from Moly Permalloy. The shields were annealed by the manufacturer. ${ }^{26} \mathrm{~A}$ 4-in.-diam hole through the end caps at one end does not appreciably deteriorate their performance. The static field is applied by means of a solenoid on an aluminum form just inside the inner shield. It is useful to have taps on the solenoid,

\footnotetext{
${ }^{25 a}$ H. P. Wills, Phys. Rev. 9, 208 (1899).

${ }^{26}$ Allegheny Ludlum Steel Corporation, Brackenridge, Pennsyl-
} 


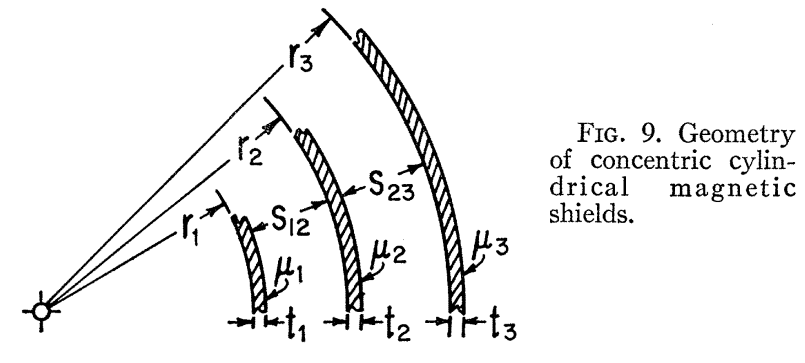

so that a few turns at each end can be energized separately. These coils can be used to reduce end effects. They are also useful for providing a small gradient for the magnetic-quenching tuning technique which will be described in Sec. X.

Proper demagnetization is critical to the shield's performance. A 60-cps demagnetizing current is passed through the center of the shields, using the vacuum can as a conductor. The resulting field is azimuthal, so that the demagnetizing flux lies completely in the shields. Current is obtained from a transformer made by winding seven turns of No. 4 copper cable around the toroidal core of a 20-A autotransformer. For fine current control, two Variacs in series are used to drive the transformer. Best results are obtained by slowly raising the current to its maximum value and then decreasing it to zero, taking about 1 min altogether.

Current up to $10^{3} \mathrm{~A}$ is required to saturate the shields, but such strong demagnetizing current is seldom necessary. The best results are obtained by demagnetizing with maximum current of about $150 \mathrm{~A}$. The residual fields appear to be caused primarily by spots of remnant magnetization in the shields. These spots vary in position and intensity from one demagnetization to another but are most commonly found along the seam of the innermost shield. The residual transverse field is generally quite nonuniform. Its maximum value is typically $10^{-5}$ to $10^{-4} \mathrm{Oe}$, based on measurements of the low-field radiation lifetime. Lower values are obtained from time to time.

By the demagnetization procedure described, very large external fields can be shielded. For instance, a powerful horseshoe magnet placed directly on the shields will cause a serious disturbance, but the disturbance is almost completely eliminated by demagnetization. Likewise, fields due to nearby large magnets on VacTon pumps can be shielded without undue difficulty. Apparently, the chief function of demagnetization is to raise the flux level to a point where the shield magnetization can favorably reorient itself.

Although large permanent fields are effectively reduced by the shields, they are much less effective at reducing small variations in the field, since the incremental permeability drops off rapidly at low fields. Addition of merely another shield does not help matters, since its shielding contribution is counterbalanced by the decrease in permeability of the inner shields which now operate at a lower field. Measurements made with a magnetometer indicate a shielding factor for small changes in the ambient field of about 600. (With the innermost shield made of $\mathrm{Mu}$ Metal, this figure is about 100.) During operation at favorable times, the field fluctuations at the storage bulb are about $10^{-7} \mathrm{Oe}$, as measured with the maser when it is operating on the field-dependent transition with a 10 -sec sampling time. The rms fluctuations are almost 10 times smaller.

\section{STORAGE BULB}

\section{A. Bulb Design}

A number of considerations are involved in designing the storage bulb. The dimensions must be chosen so that the restriction on $q$ given by Eq. (12) is satisfied, and preferably so that $q$ is as small as possible. For this reason, it is desirable to maximize the filling factor ${ }^{27}$ $\eta^{\prime}$ given by

$$
\eta^{\prime}=\frac{\left\langle H_{z}\right\rangle_{\mathrm{bulb}}{ }^{2}}{\left\langle H^{2}\right\rangle_{\text {eavity }}} \frac{V_{b}}{V_{c}} .
$$

A spherical bulb is the easiest to construct, and $\eta^{\prime}$ for that case may be determined using the plot of $\eta$ in KGR (note the correction in footnote 27). For a cylindrical bulb of radius $r$ and length $l$ in a cylindrical cavity of radius $R$ and length $L$, the following expression can be derived:

$$
\eta^{\prime}=\frac{32}{\pi^{2}} \frac{J_{1^{2}}{ }^{2}(k r)}{J_{0}^{2}(k R)} \frac{\sin ^{2}(\pi / 2)(l / L)}{\left[1+(\pi / k L)^{2}\right]} \frac{L}{l k^{2} R^{2}} .
$$

$J_{n}(x)$ is the $n$ th-order Bessel function, $J_{1}(k R)=0$. From this it follows that $\eta^{\prime}$ is maximized by the choice $r=0.52 R, \quad l=0.74 L$. Although the above formula neglects distortion of the fields in the cavity by the storage bulb, more detailed calculations show that $\eta^{\prime}$ is not significantly altered, providing the dimensions inserted in (28) refer to the actual dimensions of the cavity when it is loaded by the storage bulb. Two other considerations which enter the bulb design are the desired storage time and the collision rate. In order to avoid increasing $q$, the storage time must not be made long compared to the radiation lifetime. Because the wall shift is proportional to the collision rate, it is desirable to keep the bulb dimensions as large as possible. For this reason, a bulb somewhat larger than needed to maximize Eq. (7) may be desirable. The storage time $T_{b}$ and mean distance between wall collisions $\lambda$ are given by

$$
\begin{aligned}
T_{b} & =4 V_{b} / \bar{v} A_{a}, \\
\lambda & =4 V_{b} / A_{b} .
\end{aligned}
$$

Here $V_{b}=$ storage bulb volume, $A_{b}=$ surface area of

${ }^{27}$ The symbol $\eta^{\prime}$ is chosen since $\eta$ is the conventional nmr symbol for the filling factor. Unfortunately, $\eta$ was used in KGR for the ratio of the field averages. The vertical scale of the plot of $\eta$ in KGR has an error and should be divided by two. 
bulb, $A_{a}=$ area of the exit aperture, $v=$ average velocity $=(8 k T / \pi m)^{1 / 2}$. These formulas are derived in Appendix A. If a collimating tube is used instead of a simple aperture, then flow is reduced by a factor $K$, so that

$$
T_{b}=(1 / K)\left(4 V_{b} / \bar{v} A_{a}\right) \text {. }
$$

A table of values of $K$ for tubes of various sizes is given by Dushman..$^{2}$ A collimating tube will also slightly alter $\lambda$, and a correction for this is presented in Appendix A.

The storage bulb is generally made of fused quartz with a diameter between $3 \frac{1}{2}$ and $6 \frac{1}{2}$ in. and a wall thickness of about 0.040 in. Atoms enter the bulb through a tube whose diameter and length are chosen for the desired lifetime. If it is important to make efficient use of the beam flux, the entrance aperture can be enlarged and several thin-walled tubes introduced to make a simple collimator.

\section{B. Wall Coating}

A number of materials have been used successfully for coating the storage bulb wall. At room temperature, both long-chain paraffin ${ }^{2}$ and Dri-Film ${ }^{15}$ surfaces have been used and yield times $T_{2}{ }^{\prime}$ of about $0.3 \mathrm{sec}$ in a $16-\mathrm{cm}$ diam bulb. The limit appears to be set by the chemical reaction of hydrogen with the wall. This problem is diminished by the use of Teflon, a fluorocarbon which also has the advantage of an appreciably smaller wall shift than paraffin or Dri-Film. At $35^{\circ} \mathrm{C}$, a 16-cm-diam Teflon-coated storage bulb has a time $T_{2}{ }^{\prime}$ of abut $3 \mathrm{sec}$ and a fractional wall shift ${ }^{3}$ of $-2.1 \times 10^{-11}$. At higher temperatures, there is a decrease both in the wall shift and in the lifetime. At $100^{\circ} \mathrm{C}, T_{2}{ }^{\prime}$ is reduced by a factor of approximately 2, and the fractional wall shift is reduced by a factor of 3 . The nature of the wall relaxation is discussed in Ref. 11.

The method for applying a Teflon film has been previously reported. ${ }^{29}$ However, new techniques have been developed since then which simplify the procedure. The coating technique described in Ref. 29 is for TFE Teflon (du Pont TFE clear finish 852-201). The procedure described is improved by the use of hot white fuming nitric acid as the cleaning agent, rather than glass-cleaning solution. However, the entire coating process is simplified by the use of FEP Teflon (du Pont FEP Teflon product Code 120), since this forms a more uniform coat and several coats can be applied successively. ${ }^{11}$ The FEP suspension is applied as described in Ref. 29. However, during the fusing process clean air is circulated through the bulb. Decomposition and waste products are thereby oxidized and removed as gases. The fusing oven temperature is brought up to $360^{\circ} \mathrm{C}$ during the course of an hour, held at that temperature for about $20 \mathrm{~min}$, and then cooled, during the course of

\footnotetext{
${ }^{28}$ S. Dushman, Scientific Foundations of Vacuum Technique (John Wiley \& Sons, Inc., New York, 1962), p. 93.

${ }_{29}$ H. C. Berg and D. Kleppner, Rev. Sci. Instr. 33, 248 (1962).
}

another hour. The film is strong and transparent and is inert to hot fuming nitric acid; however, it can be removed bodily by heating in the bulb a solution of $20 \% \mathrm{HF}$ and $20 \% \mathrm{HNO}_{3}$.

The collimating tube in the neck of the bulb can be machined from solid Teflon, or it can be made by coating a pyrex plug. The latter procedure is preferable, since solid Teflon tends to outgas for several days.

Although the wall-coating procedure described here is quite reliable, the surfaces are not entirely inert, and it is possible that this is due to a contaminant in the Teflon. New surfaces are currently being investigated.

\section{ELECTRONICS}

Systems for processing the maser signal will not be described in detail, since the procedure depends on the type of measurement to be made, and often to a large extent on the available equipment. However, there are a few points of general interest.

It is important to keep the maser operating into a constant load since reactance changes will cause frequency pulling, as discussed in Sec. VI. An isolator is invaluable for this purpose. The isolator is also helpful in decoupling two masers which are operating into converters powered by a common local oscillator. Isolation requirements are stringent, if frequency locking of the masers is to be avoided. This can be seen by the following argument: The initial phase of a maser is random, since the maser originally turns on due to noise signals. The phase of the maser is constantly perturbed by thermal noise power lying within the resonance bandwidth $k T \Delta \nu_{r}$. A coherent signal of less power cannot lock the maser, since random fluctuations due to noise are sufficient to randomize the maser phase with respect to the incident signal. Therefore, isolation sufficient to reduce the unwanted signal to less than $k T \Delta \nu_{r}$ will completely prevent locking. This required 80 to $90 \mathrm{~dB}$ of isolation. Fortunately, isolators with less than $1 \mathrm{~dB}$ of insertion loss and up to $60 \mathrm{~dB}$ of isolation are available. ${ }^{30} \mathrm{It}$ is usually possible to obtain the remaining $30 \mathrm{~dB}$ of isolation through the use of balanced mixers and balanced power dividers. If the maser frequencies are offset, the isolation requirements are considerably decreased. A number of systems for processing the maser signals are described in Refs. 15, 16, 31 , and 32 .

\section{TUNING METHODS}

Tuning the maser involves setting the magnetic field to a given value, and tuning the cavity. The magnetic field is easily measured by applying a small audio signal to the bulb by a single turn of wire placed

\footnotetext{
${ }^{30}$ Ferrotec, Inc., Newton, Massachusetts; also Mel. Labs., Palo Alto, California.

${ }^{31}$ Frequency 1, 28 (1963).

${ }^{32}$ R. F. C. Vessot, H. Peters, and J. Vanier, Frequency 2,33
} 
around the cavity for that purpose. When the signal is at the Zeeman frequency, the oscillation level is markedly changed; usually the power is decreased. Alternatively, resonance of the Zeeman signal may be detected by using the fact that the hyperfine frequency is "pulled" when the Zeeman signal is close to resonance. The pulling is zero at resonance. Because the Zeeman line is as narrow as $1 \mathrm{cps}$ under favorable conditions, it is convenient to apply a standard Zeeman frequency and then trim the magnetic field to resonance.

Cavity tuning is a more involved procedure. Normally, it is done using two masers, one acting as a frequency reference while the other is tuned. The most accurate tuning methods make use of the frequency pulling effect described in Eq. (21). The resonance width is varied by a method described below, and the cavity is tuned until the maser frequency is unperturbed by variations in the linewidth.

The following two-part method has proven useful:

(1) The resonance width is altered by operating the maser under two separate conditions (which we will denote by $\mathrm{A}$ and $\mathrm{B}$ ), and the ratio $R=\Delta \nu_{A} / \Delta \nu_{B}$ is determined.

(2) The difference in pulling of the maser frequency under conditions $\mathrm{A}$ and $\mathrm{B}$ is measured. Knowledge of this figure, along with $R$, is sufficient to set the cavity to the correct frequency.

Here are details of the method:

(1) Determination of $R$. This is accomplished by offsetting the cavity frequency by some amount, typically $1 \mathrm{kc} / \mathrm{sec}$. (The actual value of the offset need not be determined.) One simple way of accomplishing this is by switching an extra current through the diode of the reactance tuner described in Sec. VI. The amount by which the frequency of the maser changes as the cavity frequency is offset is measured. We denote this quantity by $\delta . \delta$ is measured for each of the two operating conditions, A and B. From Eq. (22), it is easy to show that

$$
R=\delta_{A} / \delta_{B} .
$$

(2) Setting the cavity. The cavity tuning is now left undisturbed, and the amount by which the frequency is shifted when the maser's condition is changed from A to $\mathrm{B}$ is measured. We denote this quantity by $\Delta$.

Next, the maser is returned to condition A, and the frequency of the cavity is trimmed so that the maser frequency is increased by an amount

$$
\delta_{\text {trim }}=\Delta /(R-1) \text {. }
$$

At this point, the cavity is tuned. This can be checked by confirming that there is no frequency shift in going from $\mathrm{A}$ to $\mathrm{B}$.

Any method for varying the resonance linewidth without shifting the resonance frequency can be used for the above procedure. However, for good sensitivity, the linewidth must be changed appreciably; $R$ (or $1 / R$ ) must be large compared to 1 .

One method for varying the linewidth is to use spin exchange broadening. States A and B correspond to the maser operating near maximum and minimum flux, respectively. In this case, $R$ is close to the quantity $r$ introduced in Sec. II. [Since $R$ is known from the result of Eq. (32), this method also allows us to find the quantity parameter q.] Another method for broadening the line is to apply an inhomogeneous magnetic field, providing care is taken not to shift the average field appreciably. The latter method has the advantage of convenience, but does not afford as much sensitivity as the former method.

A third method for cavity tuning is to plot $\nu$ versus $\nu_{c}$ for different values of $\Delta \nu_{r}$, as given in Eq. (21). The result is a family of straight lines intersecting at the point $\nu_{c}=\nu_{0} .{ }^{31,33}$

An important feature of all the above methods for cavity tuning is that the effect of a spin exchange frequency shift is eliminated; the cavity will be mistuned by just the right amount to cause the maser to oscillate on the true hyperfine frequency, regardless of flux. ${ }^{3,9,32}$

How well the maser can be tuned by these or other methods depends on how far $q$ is below the critical value. Unless $q$ is substantially below the maximum permissible value, the operating range is small, and the results will not be satisfactory.

\section{ACKNOWLEDGMENTS}

The authors wish to thank the following for their contributions to the work described in this paper: A. O. McCoubrey, H. G. Robinson, E. Recknagel, E. N. Fortson, B. S. Mathur, and L. Mueller.

\section{APPENDIX: LIFETIME AND MEAN COLLISION DIS- TANCE OF ATOMS IN A STORAGE BULB}

We assume that we have an enclosed region of space (i.e., a storage bulb) in which atoms are introduced and from which they effuse through a small aperture. It is assumed for the present that the mean free path is sufficiently long and the aperture is sufficiently small so that the density of atoms throughout the bulb is constant. Let $V_{b}=$ volume of the bulb, $A_{b}=$ total area of the enclosed surface, $A_{a}=$ area of aperture, $\bar{v}=$ mean velocity of atoms, $N=$ total number of atoms in the bulb. Then the rate of loss of atoms from the bulb is $\frac{1}{4}\left(N / V_{b}\right) \bar{v} A_{a}$, and this can be shown to be equal to $N / T$, where $T$ is the mean lifetime of atoms in the bulb. Hence

$$
T=4 V_{b} / \bar{v} A_{a} .
$$

It follows that the mean distance traveled by an atom before escape is $L=T / \bar{v}=4 V_{b} / A_{a}$. However, we also

${ }^{33} \mathrm{~J}$. Vanier and R. F. C. Vessot, Appl. Phys. Letters 4, 122 (1964). 
have $L=n \lambda$, where $n$ is the mean number of collisions an atom makes before escape, and $\lambda$ is the mean distance between collisions. It can be shown that $n=\left(A_{b}+A_{a}\right) / A_{a} \simeq A_{b} / A_{a}$. Equating these two expressions for $L$ then yields

$$
\lambda=4 V_{b} / A_{b} .
$$

Application of this formula to a few simple geometries gives :

Sphere of radius $R: \lambda=4 R / 3$.

Cylinder of radius $R$ and length $L: \lambda=2 R /(1+R / 2 L)$. Cube of side $L: \lambda=2 L / 3$.
The use of a collimating tube at the bulb entrance will increase the lifetime as described by Eq. (31) in the text. It also modifies $\lambda$, since the derivation above assumes a constant density throughout the bulb. In the neck, the density falls uniformly from the equilibrium density to 0 , so that the average density is one-half that in the bulb. Since the rate of wall collision is proportional to the density, the mean wall collision rate is increased by a factor $1+\frac{1}{2}\left(V_{t} / V_{b}\right)$, where $V_{t}$ is equal to the volume of the collimating tube and we have assumed $V_{t} \ll V_{b}$. The distance between collisions is then given by

$$
\lambda=\frac{4 V_{b}}{A_{b}} \frac{1}{1+\frac{1}{2}\left(V_{t} / V_{b}\right)} .
$$

\title{
Radio-Frequency Resonance of the Metastable State $\left(2^{2} P_{3 / 2} 3^{2} S_{1 / 2}\right)_{2}$ of Neon Produced and Aligned by Electron Impact*
}

\author{
Tetsuo Hadeishi, Orilla A. McHarris, and William A. Nierenberg \\ Lawerence Radiation Laboratory, University of California, Berkeley, California \\ (Received 28 December 1964)
}

\begin{abstract}
The metastable state $\left(2^{2} P_{3 / 2} 3^{2} S_{1 / 2}\right)_{2}$ of neon was produced and aligned by electron impact. The diodestructure electron gun was operated at a high electron-current density under space-charge neutralization conditions. Radio-frequency resonance of the aligned metastable state $\left(2{ }^{2} P_{3 / 2} 3^{2} S_{1 / 2}\right)_{2}$ was observed by monitoring the change of absorption of the $\lambda 6143$-resonance radiation passing through the gap between the cathode and anode. The neon metastable-state resonance was compared with the mercury metastablestate $6^{3} P_{2}$ radio-frequency resonance obtained by using the same gun for both, and observed by monitoring the absorption of the $\lambda 5461$-resonance radiation passing through the same excitation region. The observed $\left(g_{J}\right)_{\mathrm{Ne}} /\left(g_{J}\right)_{\mathrm{Hg}}$ was $1.008 \pm 0.024$. This method could be applied to other atoms having a metastable state with $J>0$.
\end{abstract}

\section{INTRODUCTION}

A LTHOUGH the lifetime of the metastable state of noble gases such as $\mathrm{He}, \mathrm{Ne}$, and $\mathrm{Ar}$ is known to be a few milliseconds under a gas pressure of about a millimeter of $\mathrm{Hg}^{1,2}$ no optical detection of $\mathrm{rf}$ resonance other than that for helium has been reported up to the present.

One of the major difficulties in determining the rf resonance of the metastable state of the noble gases other than helium by the optical-pumping method is that their parent state has a $P$ configuration. A state having a $P$ configuration has a very fast relaxation time of the alignment at high buffer-gas pressure of about $1 \mathrm{~mm} \mathrm{Hg}$, even though the buffer gas is in the ${ }^{1} S_{0}$ ground

* This work was done under the auspices of the U. S. Atomic Energy Commission.

${ }^{1}$ A. V. Phelps and J. P. Molnar, Phys. Rev. 89, 1202 (1953), and A. V. Phelps and J. L. Pack, Rev. Mod. Phys. 26, 45 (1955).

${ }^{2}$ Tetsuo Hadeishi, Ph.D. thesis, Lawrence Radiation Laboratory Report UCRL-10477, 1962 (unpublished), and T. Hadeishi, S. S. Alpert, and W. A. Nierenberg, Bull. Am. Phys. Soc. 8, 363 (1963). state. ${ }^{3}$ This requires a very fast optical-pumping speed.

We recently reported the optical detection of $\mathrm{rf}$ resonance of the neon metastable state $\left(2^{2} P_{3 / 2} 3^{2} S_{1 / 2}\right)_{2}$ by means of production and alignment by electron impact. ${ }^{4}$ The method of producing and aligning the metastable state by high current-density electrons in fact removes the difficulties associated with the requirement for fast optical-pumping speed and a high buffergas pressure of about $1 \mathrm{~mm} \mathrm{Hg}$, since the neon metastable state is aligned during the electron transit time corresponding to about the atomic diameter. This report presents the details of the experiment.

\section{METHOD AND APPARATUS}

The neon atoms were excited to the metastable state from the ${ }^{1} S_{0}$ ground state by electron impact. The alignment due to the electron impact may be interpreted

${ }^{3}$ Peter L. Bender, Joint Institute for Laboratory Astrophysics, University of Colorado (private communication).

${ }^{4}$ Tetsuo Hadeishi, Orilla A. McHarris, and William A. Nierenberg, Bull. Am. Phys. Soc. 9, 625 (1964). 